\title{
Synthetic Quasi-TEM Meandered Transmission Lines for Compacted Microwave Integrated Circuits
}

\author{
Chih-Chiang Chen, Student Member, IEEE, and Ching-Kuang C. Tzuang, Fellow, IEEE
}

\begin{abstract}
This paper presents a two-dimensional transmission line (2-D TL) that supports quasi-TEM propagation mode and reduces problems associated with compacted meandering of microstrip (MS) on propagation constants and the characteristic impedances commonly observed in conventional one-dimensional MSs. The proposed 2-D TL comprises two layers of metallic surfaces on either side of a dielectric substrate. The top metal surface is a meandered connection of a unit cell with a central patch and connecting arms. The bottom surface is a meshed 2-D periodical ground plane, whose etched portion complements the patch portion of the top surface, forming a complementary-conducting-strip (CCS) TL, enabling a combination of an MS and MS with the tuning septa in a unit cell. Both theoretical and experimental investigations of the CCS TL agree well and demonstrate that it is much less susceptible to the effects of meanderings on the propagation constant and characteristic impedance than an MS for the same meandered pattern. Two design examples are presented to demonstrate the potential for a CCS TL for miniaturizing microwave passive circuits with minimal losses. The first example involves a 5.4-GHz CCS four-port rat-race hybrid realized in $\mathrm{RO4003}$ and reduces the area of original MS design by $87 \%$. The second example illustrates the applicability of a CCS TL to a monolithic RF integrated circuit using a first-pass design of a 5.2-GHz CMOS oscillator incorporating a CCS TL as a resonator with an area totaling $500 \times 600 \mu \mathrm{m}^{2}$ including pads base on Taiwan Semiconductor Manufacturing Company's 0.25- $\mu \mathrm{m}$ 1P5M CMOS process techniques.
\end{abstract}

Index Terms-Quasi-TEM, rat-race hybrid, synthetic guiding structure, transmission line (TL).

\section{INTRODUCTION}

$\mathbf{P}$ ROLIFERATION of wireless devices into daily human lives has been a global phenomenon. One key factor for such success of wide acceptance of wireless products is the miniaturization of wireless apparatus, thanks to the continuing improvement of semiconductor processes, shrinking photolithography, and multilayered integration technologies such as the low-temperature co-fired ceramics (LTCCs) and printed circuit boards (PCBs) [1]-[3]. A variety of methods for shrinking microwave devices have been reported. Small microwave ceramic components often employ high relative dielectric constant $\left(\varepsilon_{r}\right)$ substrates, sometimes $\varepsilon_{r}$ reaching 100

Manuscript received December 3, 2003; revised February 9, 2004. This work was supported in parts by the National Science Council of Taiwan under Contract NSC92-2213-E-009-020 and by the Academic Excellence Program under Contract 89-E-FA06-2-4.

C.-C. Chen is with the Institute of Communication Engineering, National Chiao Tung University, Hsinchu, Taiwan, R.O.C.

C.-K. C. Tzuang is with the Graduate Institute of Communication Engineering, Department of Electrical Engineering, National Taiwan University, Taipei, Taiwan, R.O.C. (e-mail: cktzuang@cc.ee.ntu.edu.tw).

Digital Object Identifier 10.1109/TMTT.2004.828468
[4]-[6]. The device dimension is typically inversely proportional to the square root of $\varepsilon_{r}$ [7]. An alternative to applying high $\varepsilon_{r}$ materials is the use of high-permeability materials for miniaturization [8], [9]. Slow-wave guiding structures, on the other hand, have been extensively studied for making smaller low-loss passive components, e.g., the uniplanar compact photonic-bandgap (UC-PBG) filters [10] and electric-magnetic-electric (EME) filters [11], which apply the periodical structures in either the ground plane or signal path to increase the slow-wave factor (SWF) of basically the microstrip (MS) structures.

Without resorting to the high-permittivity and high-permeability materials or the exotic periodical structures for making smaller microwave devices, thin-film microstrips (TFMSs) has been a popular technique based on the scaling of microwave guiding structure at the cost of much higher losses encountered during the scaling-down process [12]-[17]. TFMSs are typically fabricated above the active monolithic microwave integrated circuit (MMIC) devices with multilayered metal interconnects, in which various forms of planar or quasi-planar transmission lines (TLs) can be made [14]-[17]. It has been shown that the losses of the TFMSs are inversely proportional to the substrate thickness and proportional to surface resistivity [12]. To save chip area, designers often meander the TFMSs, thus making very compact passive components in the MMICs [12]-[15], [18], [19] and hybrid microwave integrated circuits (HMICs) [20], [21]. For example, a wide-band 6-18-GHz monolithic magic-T occupying chip size of $0.9 \mathrm{~mm} \times 1.0 \mathrm{~mm}$ was reported, which included two quarter-wavelength TFMSs, and transitions of slotline-to-TFMS $(0.2 \mathrm{~mm} \times 0.4 \mathrm{~mm}$ each $)$ and TFMS-to-coplanar waveguide (CPW) [13]. The two quarter-wavelength TFMSs occupy most of the chip area, approximately $0.3 \mathrm{~mm} \times 0.5 \mathrm{~mm}$. The first reason for such successful miniaturized design lies in the fact that the TFMS $50-\Omega$ TLs can be fabricated on a thin 3- $\mu \mathrm{m}$ silicon oxynitride (SiON) substrate of width $5 \mu \mathrm{m}$ and pitch $15 \mu \mathrm{m}$ for the adjacent TFMSs. The second reason, which is less noticeable, is the use of meandered TFMS structure. This makes the quarter-wavelength TFMS very compact in area.

There are, however, two drawbacks of applying the meandered TFMSs. First, the linewidth $W$ of the TFMS is the single control parameter for varying the characteristic impedance $Z_{c}$ once process parameters such as the substrate thickness $(h)$ and the dielectric material are decided. This places a strict limitation over the use of a high-impedance line since the MS will become unrealistically narrow. Furthermore, many microwave hybrids and passive circuits such as couplers, filters, and power dividers mandate TLs of wide-range characteristic impedance [22]-[24]. 
Consequently, designing TFMS passive and hybrid circuits is a challenging issue. Second, meanderings of the MS drastically alters the propagation characteristics of the bound $E H_{0}$ mode in phase constant $\beta$ and characteristic impedance $Z_{c}$. Such drastic changes of $Z_{c}$ and $\beta$ are the result of coupling among the nearby TFMSs and the right-corner bends of the meandered lines. Thus, a larger ratio of adjacent lines spacing $\left(l_{g}\right)$ to substrate height (h), typically greater than two [12], is applied to avoid serious degradation in guiding characteristics. This, however, adversely affects the efforts for making miniaturized microwave circuits.

This paper proposes a two-dimensional transmission-line (2-D TL) concept advancing the art of conventional MS lines, which are one-dimensional. Being one-dimensional, the conventional MS guiding structure is uniform in cross section along the guiding direction. The proposed 2-D synthetic TL is not uniform in cross section along the propagating axial direction. Such an additional degree of freedom in the guiding structure makes the equivalent quasi-TEM $E H_{0}$ mode of propagation able to synthesize a wide range of characteristic impedances and place passive TL circuits in a compact area concurrently.

Section II qualitatively describes how the synthetic guiding structure works. Section II also introduces a figure-of-merit called the area reduction factor (ARF) for explaining how the synthetic 2-D TL significantly reduces the size of a four-port hybrid by employing the proposed guiding structure against the conventional MS. Section III investigates the guiding characteristics of the proposed guiding structure meandered in a 2-D periodical surface, showing much wider range of characteristic impedance necessary for designing TL circuits as opposed to those realized by MS meandered in the same way. Section IV shows a design example for making a miniaturized rat-race HMIC using the synthetic TL designs reported in Section III. Section V investigates the applicability of the synthetic TL replacing the lumped $L C$ resonators in a monolithic RF CMOS oscillator, demonstrating the potential use of the synthetic TL in RF integrated circuits (RFICs). Section VI concludes this paper.

\section{OPERATIONAL PRINCIPLE OF MEANDER COMPLEMENTARY CONDUCTING STRIPS (CCSs)}

This section qualitatively describes the principle of operation of the synthetic TL proposed by this study, which is particularly suitable for the syntheses of quasi-TEM TLs of a broader characteristic impedance range than that of the conventional MS to carry on high-performance microwave passive circuit designs. The proposed guiding structures are made on multilayered substrates such as PCBs, LTCCs, and MMICs like RF CMOS and GaAs pseudomorphic high electron-mobility transistor (pHEMT) processes. Following the experiences of designing a broad range of miniaturized microwave integrated circuits using the proposed TL structures, this paper presents the basic design concepts, guiding characteristics, and some important application examples for illuminating the ideas of making a fundamentally 2-D quasi-TEM guiding structure that offers an additional one degree of freedom for making controlled impedance lines to meet design needs.

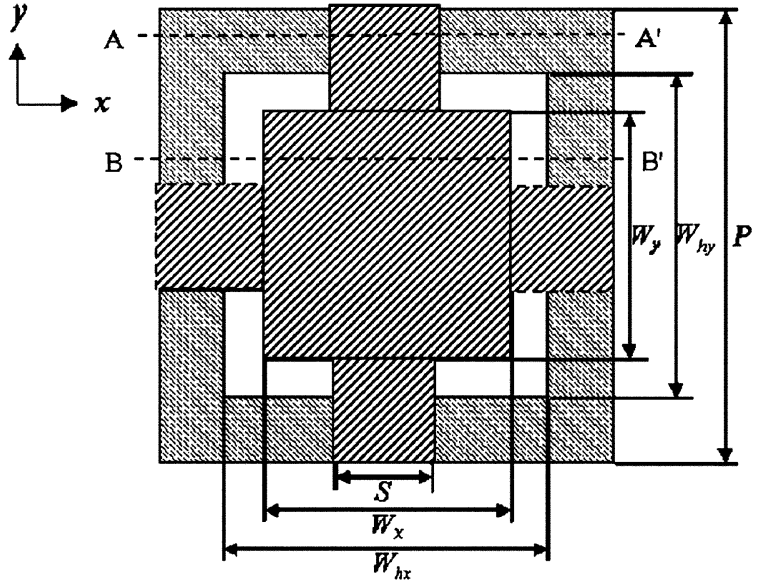

(a) Two-dimensional (2-D) top view

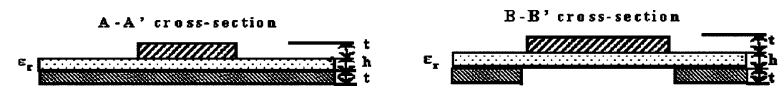

(b) Cross-section view for the MIC

Fig. 1. Geometries of the CCS unit cell. (a) 2-D top view. (b) Cross-sectional view.

\section{A. Proposed Concept of Quasi-TEM Synthetic TL}

The proposed quasi-TEM TL is a one-dimensional periodical structure meandered in a 2-D plane. A typical unit cell, shown in Fig. 1(a), has dimensions much smaller than the operating wavelength $(\lambda g)$, usually 20 cells in a quarter-wavelength line. In the unit cell of Fig. 1, the top metal layer consists of a patch with four connecting arms for four-way interconnects, in which at least two arms are employed for connections of cells. At the bottom of the unit cell is the mesh ground plane structure, which forms a 2-D periodical structure for the ground plane of the proposed guiding structure. Consider a square periodical structure with a periodicity of $P$. The guiding structure integrates two TLs in a single cell by drawing $A-A^{\prime}$ and $B-B^{\prime}$ cuts horizontally across the unit cell. The left column of Fig. 1(b) displays a cross-sectional view of the unit cell along the $A-A^{\prime}$ cut for hybrid PCB/MMIC and lower Si or GaAs substrate without. The left-hand-side column clearly shows that the well-known MS structure is independent of the passivation in the MMIC process. The right-hand-side column in Fig. 1(b), however, presents an MS with the tuning septa [25] or, equivalently, an elevated CPW [26] across the $B-B^{\prime}$ cut of the unit cell. Varying the width of the patch $W_{x}\left(W_{y}\right)$ and the inner hole dimension of the mesh $W_{h x}\left(W_{h y}\right)$, the high-to-low characteristic impedance ratio of 10.1 had been reported for the MS with the tuning septa [25]. On the other hand, the practical range of tuning the characteristic impedance of a conventional MS by varying the width $S$ is typically much less than that of the MS with the tuning septa, say, 8.0. As the guided electromagnetic wave traverse the unit cells, it experiences the alternating types of guiding structures of different characteristic impedance levels in distances far less than the equivalent wavelength of the resultant TL. Since an MS with and without the tuning septa support the quasi-TEM mode and discontinuities inherent in the unit cell are comparably small against the equivalent wavelength, the combined guiding structure of the unit cell also sup- 


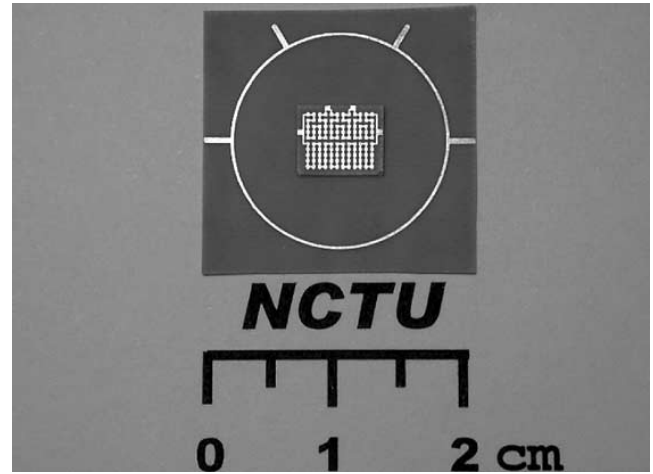

Fig. 2. Comparison of mask patterns of the 2-D guided structures of the CCS rat-race hybrid prototype (inside pattern) and the traditional MS rat-race hybrid (outside pattern).

ports the quasi-TEM mode by merging two types of guiding structures. In other words, electrical fields emanating from the positive charges on a top signal trace should always find the shortest path to terminate at the bottom conductive strips in a very similar manner as those of an MS with and without the tuning septa, thus rendering the quasi-TEM mode, provided the dimension $P$ of the unit cell is much smaller than the operating wavelength. In this case, the discontinuities associated with the proposed guiding structure will not cause significant radiation losses or stopband characteristics frequently typically observed in the periodical structures. This paper, however, focuses only on the quasi-TEM propagation characteristics of the CCS MS in the lower frequency region, where many useful applications are desirable.

The patch of dimensions $W_{x}$ by $W_{y}$ and the mesh ground plane with hole of dimensions $W_{h x}$ by $W_{h y}$ form complementary surfaces, therefore, we call the proposed TL the CCS TL. The upper metallic patch surface is connected to the adjacent cells by connecting arms, which form a relatively small overlapping area to both top and bottom surfaces and slightly perturb the complementary surfaces. In Section III, we will demonstrate that, given the same periodicity $P$ and the fabrication process, various CCS TLs can be synthesized to yield the same characteristic impedance. Such a unique property of the CCS TL is a manifestation of the fact that the proposed quasi-TEM guiding structure is a 2-D structure in view of its periodical variations along the propagating direction while maintaining the same substrate geometry, thus supporting an additional degree of freedom for designing TLs.

\section{B. Compacted Passive Circuits Using CCS TLs}

The most important feature of the proposed CCS TL is its application for designing compacted TL passive circuits such as the one shown in Fig. 2, which is a photograph of the experimental 5.4-GHz rat-race hybrids designed by employing conventional MS and the CCS TL, respectively, to achieving nearly the same four-port network parameters. Both designs are printed simultaneously on a Roger RO-4003 0.203-mm substrate of relative dielectric constant $\varepsilon_{r}$ equal to 3.38. One immediately recognizes that the compacted rat-race hybrid based on the CCS TLs occupies much less area than the familiar ring-shaped design using a conventional MS, which wastes a substantial area by over $80 \%$. Section III will show why the CCS TL is much better than MS in guided-wave propagation characteristics for compacted meandered microwave passive circuit designs. Here, we report the basics necessary for an accurate assessment of area size and make a comparison between the designs using the conventional MSs and CCS TLs before entering the serious design phase. Given the case study of the $5.4-\mathrm{GHz}$ four-port rat-race hybrid, a total wavelength of $6 / 4 \lambda g$ is required for both designs using MSs and CCS TLs. In the typical ring-shaped configuration of a rat-race hybrid based MS, the circumference of the ring is

$$
\left(\frac{6}{4}\right) \lambda_{g 1}=2 \pi R_{1}
$$

where $R_{1}$ is the radius of the ring and $\lambda_{g 1}$ is the guided wavelength of the conventional $70-\Omega$ MS line at the operating frequency $f_{o}$. Excluding the T-junctions required for the four-port interface, the estimated area of an MS rat-race hybrid $(A 1)$ is

$$
A_{1}=\pi R_{1}^{2}=\left(\frac{9}{16 \pi}\right) \lambda_{g 1}^{2}
$$

The CCS TL realization of microwave passive circuits adopts an entirely different philosophy by placing the meandered CCS TLs in a compacted 2-D plane in an array shape by simply connecting the connecting arms of the cells along the desired directions of propagation. Thus, the total area $(A 2)$ required to accomplishing the design example of the rat-race hybrid is

$$
A_{2}=\left(\frac{6}{4}\right) \lambda_{g 2} \cdot P
$$

To this end, an ARF of the particular case study can be expressed by

$$
\mathrm{ARF}=1-\frac{A_{2}}{A_{1}}=1-\frac{\left(\frac{8 \pi}{3}\right)\left(\frac{P}{\lambda_{o}}\right)\left(\mathrm{SWF}_{\mathrm{MS}}\right)^{2}}{\mathrm{SWF}_{\mathrm{CCS} \mathrm{TL}}}
$$

where SWFs of the MS and CCS TL are, respectively, defined as $\mathrm{SWF}_{\mathrm{MS}}=\lambda_{\mathrm{o}} / \lambda_{\mathrm{g} 1}$ and $\mathrm{SWF}_{\mathrm{CCS} \mathrm{TL}}=\lambda_{\circ} / \lambda_{g 2}$, and $\lambda_{o}$ is the guided wavelength in free space at the operating frequency $f_{o}$. Equation (4) shows a linear dependence of the ARF against the periodicity $P$ of the CCS TLs. The smaller the value of $P$ will result in a larger ARF, which is a figure-of-merit to demonstrate the important area of CCS TLs in the process of designing compacted microwave circuits. The proper choice of $P$ for making CCS TLs depends upon the following three factors:

1) required range of the characteristic impedances of the TL;

2) minimum linewidth and line spacing of the particular integrated-circuit process;

3) maximum current handling capability of the CCS TL Quick estimation by assuming that $\mathrm{SWF}_{\mathrm{MS}}=\mathrm{SWF}_{\mathrm{CCS}} \mathrm{TL}=$ $1.5, P=500 \mu \mathrm{m}$, and $f_{0}=5.4 \mathrm{GHz}$, the ARF will be $88.7 \%$, which is very close to detailed analyses and experimental results to be discussed. 


\section{QUASI-TEM PROPAGATION CHARACTERISTICS OF MEANDERED CCS TLs AND CONVENTIONAL MSs}

\section{A. Analyses and Validity Checks of 2-D TLs}

As discussed in Section II, the CCS TL is a 2-D guiding structure since it is not uniform in the cross sections along the direction of propagation [see Fig. 1(b)]. Furthermore, the CCS TL may traverse in an arbitrarily meandered path. Full-wave resonance techniques for extracting the quasi-TEM propagation characteristics may not apply. What follows summarizes the procedure for extracting the effective complex propagation constant $\gamma$ and the complex characteristic impedance $Z_{c}$ from the theoretical and measured two-port scattering parameters. The effective complex propagation constant $\gamma$ is denoted by $\gamma=j \beta+\alpha$, where $\beta$ is the phase constant and $\alpha$ is the attenuation constant. Using the $A B C D$ matrix representation of a two-port TL in terms of complex propagation constant $\gamma$ and characteristic impedance $Z_{c}$, and the link between the $A B C D$ matrix and the scattering parameters ( $S$-parameters), one obtain the following equations [27]:

$$
\begin{aligned}
e^{\gamma L} & =\frac{1-S_{11}^{2}+S_{21}^{2}+\sqrt{\left(1+S_{11}^{2}-S_{21}^{2}\right)^{2}-\left(2 S_{11}\right)^{2}}}{2 S_{21}} \\
Z_{c} & =Z_{o} \sqrt{\frac{\left(1+S_{11}\right)^{2}-S_{21}^{2}}{\left(1-S_{11}\right)^{2}-S_{21}^{2}}}
\end{aligned}
$$

where $Z_{o}$ is the referenced impedance, typically equal to $50 \Omega$; $L$ is the total length of the meandered CCS TL, equal to $P$ times the number of cells. Throughout this paper, the full-wave simulator Zeland IE3D is employed for obtaining the two-port scattering parameters of the meandered TLs. Metal strip conductivity of $5.49 \times 10^{7} \mathrm{~S} / \mathrm{m}$ and a thickness of $18 \mu \mathrm{m}$ (approximately a 1/2-oz copper thickness), and the dielectric loss tangent of 0.002 are entered in the full-wave analyses.

Referring to Fig. 2, the HMICs are made on a two-sided printed RO4003 circuit board of thickness $(h) 0.203 \mathrm{~mm}$ and relative permittivity $\left(\varepsilon_{r}\right) 3.38$. For the purpose of clarity, we present the propagation characteristics of quasi-TEM TLs by characterizing the TLs in terms of the SWF (SWF $=\lambda_{o} / \lambda_{g}=\beta / k_{o}$ or the normalized phase constant) and the characteristic impedance $\left(Z_{c}(f)\right)$, both of which are dispersive. The losses associated with the guiding structure are expressed by decibels $/ \lambda_{g}$. All three parameters, namely, $Z_{c}, \mathrm{SWF}$, and loss (decibels $/ \lambda_{g}$ ), are readily derived from (5) and (6).

The validity checks of the above TL parameter-extraction procedure is conducted by comparing measured results and theoretical data using the same test circuit, which is shown in the inset of Fig. 3, i.e., a CCS TL meandered in the $5 \times 42$-D lattice. The CCS TL employs a unit cell of periodicity $P$ equal to $450 \mu \mathrm{m}$, a top metal patch of dimensions $W_{x}=W_{y}=300 \mu \mathrm{m}$, a connecting arm of width $S=150 \mu \mathrm{m}$, and a square opening in the mesh ground plane of $W_{h x}=W_{h y}=300 \mu \mathrm{m}$. Fig. 3 plots both theoretical (in solid lines) and measured (in dotted solid lines) data, showing that $3.73 \%$ deviation in the normal-

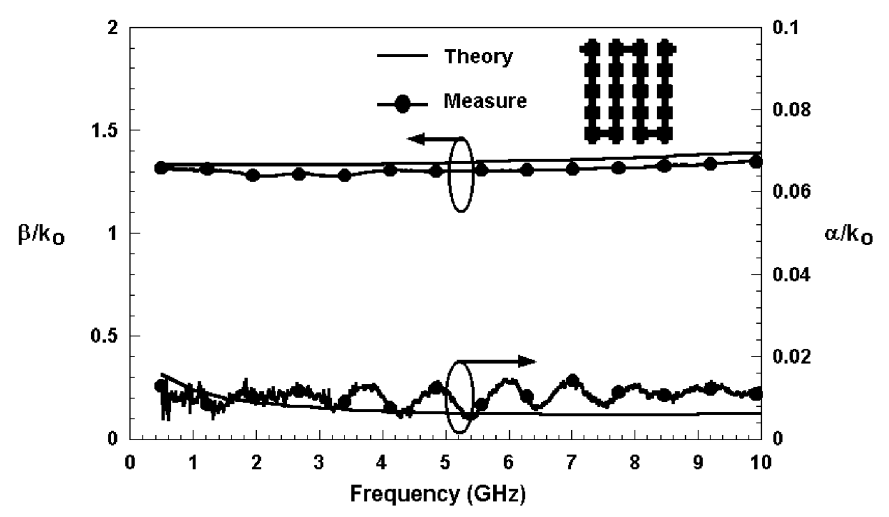

Fig. 3. Predicted and measured normalized propagation constants (phase constant $\beta / k_{o}$, attenuation constant $\alpha / k_{o}$ ) versus frequency of the 70- $\Omega$ CCS TL $(5 \times 4$ meander trace $)$. Structural and material parameters are $P=450 \mu \mathrm{m}$, $S=150 \mu \mathrm{m}, W_{x}=W_{y}=W_{h x}=W_{h y}=300 \mu \mathrm{m}, L=9020 \mu \mathrm{m}$, and $h=0.203 \mathrm{~mm}$; the relative dielectric constant $\varepsilon_{r}=3.38$ and $t=0.018 \mathrm{~mm}$, and the conductivity of the metal is $5.49 \times 10^{7} \mathrm{~S} / \mathrm{m}$, as shown in Fig. 1 and (5).

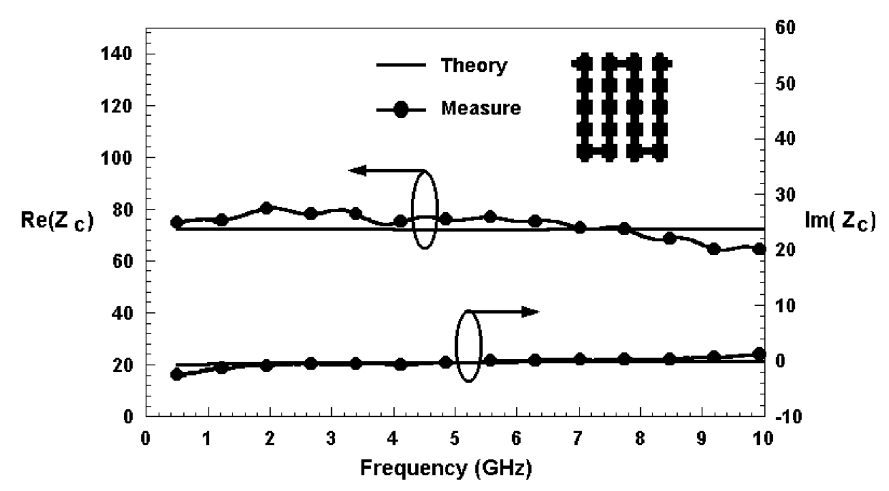

Fig. 4. Predicted and measured characteristic impedance (real part: $\operatorname{Re}\left(Z_{c}\right)$, imaginary part: $\left.\operatorname{Im}\left(Z_{c}\right)\right)$ versus frequency of the $70-\Omega \operatorname{CCS}$ TL $(5 \times 4$ meander trace); structural and material parameters as in Fig. 3.

ized propagation constant and the theoretical values of the normalized attenuation constant $\left(\alpha / k_{o}\right)$ are very close to the lower bound of the measured data. The two sets of curves are in excellent agreement across the entire spectrum of interest from 0.5 to $10 \mathrm{GHz}$. Thus, confidence in the theoretical extractions of propagation characteristics of the compacted meandered CCS TL is established. The discrepancy in complex $\gamma$ could be caused by a small amount of internal multiple reflections since two subminiature A (SMA) connectors are soldered to the CCS TL in the test fixture for reliable $S$-parameters testing and the residual reflections are still present after deembedding the test fixture.

Fig. 4 compares the complex characteristic impedances obtained by extracting theoretical and measured scattering parameters for the same CCS TL reported in Fig. 3. The theoretical value of $72 \Omega$ is slightly lower than that of measured data near $5.4 \mathrm{GHz}$. Across the $0.5-10-\mathrm{GHz}$ band, the maximum (minimum) value of the real part of the characteristic impedance is $80(64) \Omega$, representing a deviation of $+/-11 \%$ from $72 \Omega$. The imaginary parts of both theoretical and measured complex characteristic impedances are nearly identical, except near the beginning and ending frequencies. The data shown in Fig. 4 also validate the theoretical predictions of the guiding characteristics of the compacted meandered CCS TL. 


\section{B. Comparative Studies of Propagation Characteristics of CCS TL Against MS in Both Straight and Meandered Configurations}

In the following, the propagation characteristics of a 2-D CCS TL as previously shown in the inset of Fig. 3 will be investigated by varying the planar structural parameters and comparing the extracted theoretical data of complex propagation constants and characteristic impedances against those of conventional MSs in both straight and meandered shapes. Assuming that all the guiding structures under investigations are made by the same process and design rules such that the minimum linewidth is $100 \mu \mathrm{m}$, the minimum line spacing is $100 \mu \mathrm{m}$, and the material constants are the same as those applied for the CCS TL design reported in Figs. 3 and 4. The guiding properties $\operatorname{Re}\left(Z_{c}\right)$ (real part $\left.Z_{c}\right)$, SWF, and $\operatorname{Loss}\left(\mathrm{dB} / \lambda_{g}\right)$ of the 2 -D CCS TL $(5 \times 4$ meander trace) versus different values of patch width $W$ (= $\left.W_{x}=W_{y}\right)$ under the conditions of various widths $(S)$ of the connecting arm and changing sizes of etched ground planes $W_{h}$ $\left(=W_{h x}=W_{h y}\right)$ and the periodicity $P$ of 450 and $225 \mu \mathrm{m}$ at $f_{o}=5.4 \mathrm{GHz}$ are displayed in Fig. 5(a)-(c), respectively.

This section investigates the guiding properties of a CCS TL realized by contemporary $\mathrm{PCB}$ process obeying design rules generally accepted by most manufacturers. Such investigations lead to the design of the compacted rat-race hybrid shown in Fig. 2. Useful design information and comparative studies between the conventional MS and synthetic CCL TL are presented with emphasis on effects of meanderings on guiding characteristics.

Assuming that RO4003 substrate of thickness of $0.203 \mathrm{~mm}$ and relative dielectric constant of 3.38 is employed for the synthetic CCS TL design, we begin with a straight-line configuration for both conventional and synthetic MS investigations. Fig. 5(a) plots the real part of the deembedded characteristic impedances against the width $(W)$ of MSs. The lower bound of the width $W$ is $100 \mu \mathrm{m}$, reflecting the typical limit of the present PCB foundry design rules. The upper bound is set to $350 \mu \mathrm{m}$, allowing room for the design of a high-impedance synthetic CCS TL.

The characteristic impedance of a straight CCS TL, in the L1 cell form, is $9.43 \%, 28.75 \%$, and $27.83 \%$ higher than that of a straight MS line at $W=100,200$, and $350 \mu \mathrm{m}$, respectively. For the particular case of the straight CCS TL, the width of connecting arm $S$ and the dimension of a square ground plane opening $W_{h}$ are 100 and $350 \mu \mathrm{m}$, respectively. The combined result of alternating: 1) the MS and 2) the MS with the tuning septa produce the effective characteristic impedance, which are deembedded from the theoretical end-to-end two-port scattering parameters of the overall CCS TL, which is much greater than the conventional straight MS line.

The results of the second comparative study are also superimposed to the previous study, namely, the meandered MS and the meandered synthetic CCS TL following the same winding course. The plot located in the lowest level of Fig. 5(a) is the change of characteristic impedance of a meandered MS in the course of a $5 \times 4$ matrix against the width of the patch. Notice that the meandered arrangement of the MS significantly reduces the characteristic impedance by approximately 8.7 to $21.24 \%$ across the range of width variation from 100 to $350 \mu \mathrm{m}$

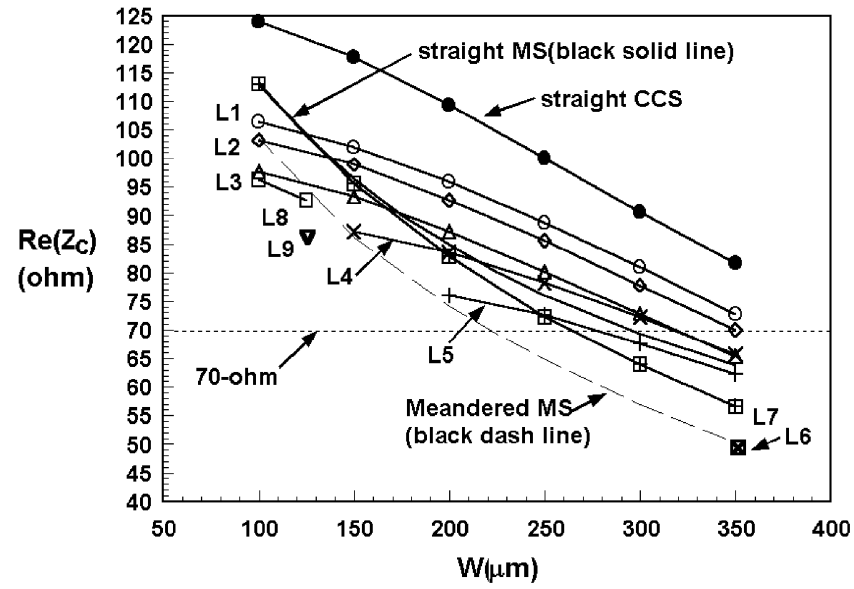

(a) The $\operatorname{Re}\left(Z_{c}\right)$ vs. W

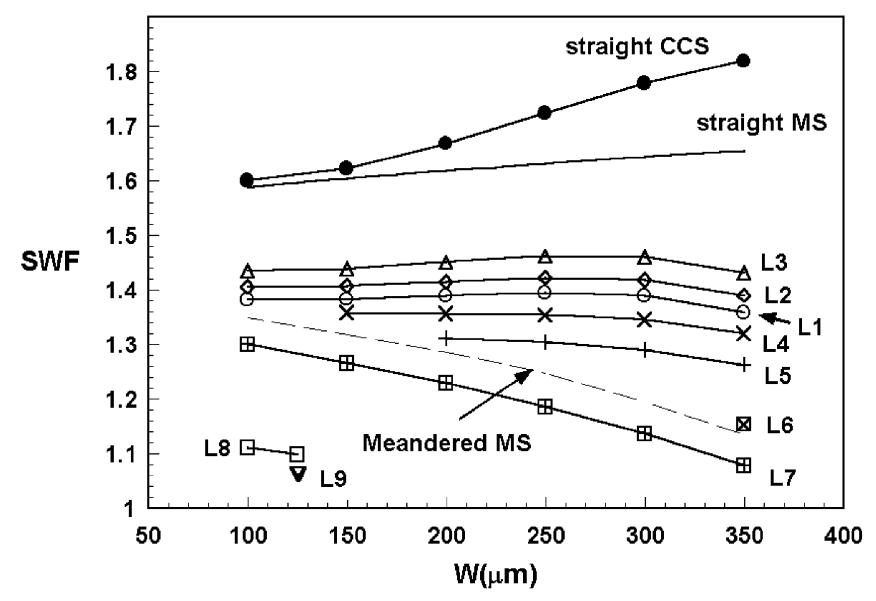

(b) The SWF vs. W

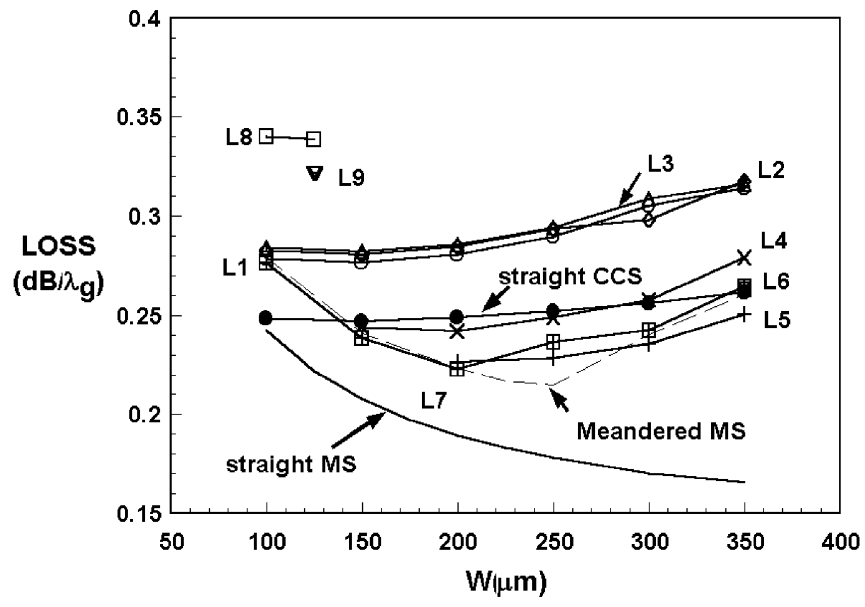

(c) The $\operatorname{loss}\left(\mathrm{dB} / \lambda_{\mathrm{g}}\right)$ vs. W

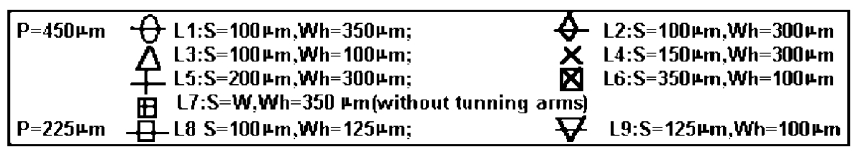

Fig. 5. Comparison of guiding properties. (a) $\operatorname{Re}\left(Z_{c}\right)$, (b) SWF, and (c) loss per guided wavelength (decibels $/ \lambda_{g}$ ) of the CCS TL and MS in both straight and meandered configurations $(5 \times 4$ meander trace $)$ versus patch width $W(=$ $\left.W_{x}=W_{y}\right)$ given various widths $(S)$ of the connecting arm and various sizes of etched ground planes $W_{h}\left(=W_{h x}=W_{h y}\right)$; periodicity $P=450$ and $225 \mu \mathrm{m}$ at $f_{o}=5.4 \mathrm{GHz}$.

in the particular case study. The corresponding meandered synthetic CCS line, marked by L1, shows the variation of $Z_{c}$ from 
106.4 to $72.8 \Omega$ in contrast to the impedance variation from 103.4 to $50.3 \Omega$ observed in the meandered MS. This particular case study clearly indicates that the synthetic CCS TL is less susceptible to meanderings than MS. Notice that periodicity $(P)$ of $450 \mu \mathrm{m}$ is assumed in this particular case study. When $W=350 \mu \mathrm{m}$, the minimum spacing between two meandered MSs (synthetic CCS TL) is only $100 \mu \mathrm{m}$, which is one-half of the substrate height, the adjacent lines in the meandered $5 \times 4$ matrix must couple strongly. Nevertheless, the CCS TL shows a stronger resistance to lowering the characteristic impedance by meanderings, thus, the slope of the $L_{1}$ curve is much flatter than that of the black dashed line of the meandered MS.

The third comparative study compares the variations characteristic impedances within the meandered CCS TLs by varying the ground-plane openings from $W_{h}=350 \mu \mathrm{m}$ to $W_{h}=$ $100 \mu \mathrm{m}$ while maintaining the same width of the connecting arm at $S=100 \mu \mathrm{m}$. As expected, reducing the size of the ground-plane opening or, equivalently narrowing the gap of the tuning septum, the characteristic impedance should be lower [25]. The results, marked by L1, L2, and L3 with symbols in a hollow circle $(0)$, hollow rhombus $(\diamond)$, and triangle, successively decrease in values at an approximately equal increment as $W$ varies. The lowest $Z_{c}$ of these three plots occur for L3 at $W=350 \mu \mathrm{m}$, implying that a small perturbation in the ground plane by etching a square hole of $100 \mu \mathrm{m} \times 100 \mu \mathrm{m}$ can still result in a much higher $Z_{c}$ of $65.3 \Omega, 30 \%$ greater than that of a meandered MS. In the third comparative study, the L1 curve (with $W_{h}=350 \mu \mathrm{m}$ ) shows the highest value in $Z_{c}$.

The fourth comparative study investigates the effect of $S$ (width of the connecting arm of the CCS cell) on $Z_{c}$ for periodicity $P$ equal to $450 \mu \mathrm{m}$ and a ground-plane square hole opening of $300 \times 300 \mu \mathrm{m}$. This is equivalent to saying that, beginning with the L 2 curve (connected by rhombus symbols), we increase the value of $S$ from $100 \mu \mathrm{m}$ through $150 \mu \mathrm{m}$ to $200 \mu \mathrm{m}$. The results are represented by $\mathrm{L} 2, \mathrm{~L} 4$, and $\mathrm{L} 5$, respectively. To our expectation again, an increase in $S$ results in a wider MS section of the CCS TL, therefore, lowering $Z_{c}$. Consequently, L5 shows the lowest $Z_{c}$ in this comparative study. L2, L4, and L5 stretch out to the right for $W=100$, 150 , and $200 \mu \mathrm{m}$, respectively. Notice that the left-hand-side starting points of L2, L4, and L5 form an asymptote that is very close to the plot of the meandered MS. Such observation indicates that the CCS TL with $S=W$ and $W_{h}=300 \mu \mathrm{m}$ has similar $Z_{c}$ to that of the meandered MS, implying that $W_{h}$ is not big enough for making an impact on guiding properties.

If we decrease the value of $W_{h}$ further to $100 \mu \mathrm{m}$, with anticipation that the meandered CCS TL behaves like a meandered MS, the results are plotted as a single point L6 for $S=350 \mu \mathrm{m}$, clearly showing that L6 is on the same $Z_{c}$ plot of the meandered MS. If we increase the value of $W_{h}$ from 300 to $350 \mu \mathrm{m}$ by merely $50 \mu \mathrm{m}$ and keep $S=W$, the resultant plot L7 shifts upwardly from the asymptote or the $Z_{c}$ plot of the meandered MS, implying that the size of the ground-plane opening is large enough to make a noticeable effect on guiding characteristics.

The last and sixth comparative study is based on a smaller cell size of $P$ equal to $225 \mu \mathrm{m}$. Limited by the PCB design rules, the permissible tuning range is much smaller. Assuming that $W_{h}=125 \mu \mathrm{m}$, the results are shown in plot L8 for $W$ between 100-125 $\mu \mathrm{m}$. When compared to plots L1, L2, and L3 that incorporate design rules of $S=100 \mu \mathrm{m}$ and $P=450 \mu \mathrm{m}$, one observes that use of a smaller cell may pay the penalty for smaller $Z_{c}$. At $W=100 \mu \mathrm{m}$, the highest value of $Z_{c}$ of L8 is $96.3 \Omega$, considerably smaller than those that appear in L7, L1, and $\mathrm{L} 2$.

Fig. 5(b) plots the SWF of all case studies reported in Fig. 5(a) against the variation of width $(W)$ in the MS or CCS TL. One immediately recognizes that meanderings of TLs also cause a significant reduction in the SWF. For the particular MS, the effect of meanderings on a slow-wave reduction can be as high as $15.02 \%$ for $W=100 \mu \mathrm{m}$ and $31.45 \%$ for $W=350 \mu \mathrm{m}$. Case studies of L1-L5, which exhibit higher resilience to the effect of meandering on a change of $Z_{c}$, also display relatively flat curves. Comparing cases L1-L3, increasing $W_{h}$ (the size of the opening in the ground plane) results in a decrease in the SWF and an increase in $Z_{c}$. Examining L4 and L5, an increase of $S$ (the width of connecting arms) reduces $Z_{c}$ and SWF simultaneously. The mechanism by which the SWF decreases by increasing $S$ reduces the SWF and mainly involves a fall in the equivalent inductive component of the stepped MS [28]. The meandered CCS TL of equal $S$ and $W$, however, displays a constant downward shift from the plot for the meandered MS, reflecting the fact that the ground-plane openings actually decrease the SWF, as in the case studies of L1-L3 have demonstrated. When $P=225 \mu \mathrm{m}$, half of the previous case studies of L1-L7, the SWF is near 1.1 or below, as observed in L8 and L9. The reduction in cell size (periodicity) $P$ in this particular case study adversely affects the SWF of the meandered CCS TL. Care should be exercised as to not deteriorate the SWF below the lower bound limit of the meandered MS by properly designing a correct configuration of the CCS TL.

Fig. 5(c) reports the attenuation constant of the various TL designs in units of losses in decibels per guided wavelength $\left(\mathrm{dB} / \lambda_{g}\right)$. Since the SWFs of the meandered MS and CCS TL are lower than the straight MS (or straight CCS TL), one may speculate that the attenuation constant in decibels per guiding wavelength should be highest for L8 and L9, followed by L7, L5, L4, L1, L2, L3, etc., as observed in Fig. 5(b). This is, however, not the case of simply reversing the order of Fig. 5(b). Fig. 5(c) shows that the straight MS line has the lowest value in loss for the entire range of $W$ of interest. The meandered MS, which shows higher attenuation as expected, appears approximately having the lower bound attenuation constants to all meandered CCS TLs under investigation. Of more interest is the fact that the lowest attenuation constants of all case studies L1-L6 form an asymptote that is nearly the replica of that for the meandered MS. All case studies reported in Fig. 5(c) demonstrate that the attenuation constants are generally less than $0.35 \mathrm{~dB}$ per guided wavelength, which is a practical value for most distributed microwave-circuit designs.

\section{Dispersion Characteristics of 2-D TLs}

The dispersion characteristics of meandered TLs should be investigated before completing the comprehensive studies of the synthetic TLs. In Fig. 5, all results are focused on a single frequency at $5.4 \mathrm{GHz}$. This section pays attention to a specific 


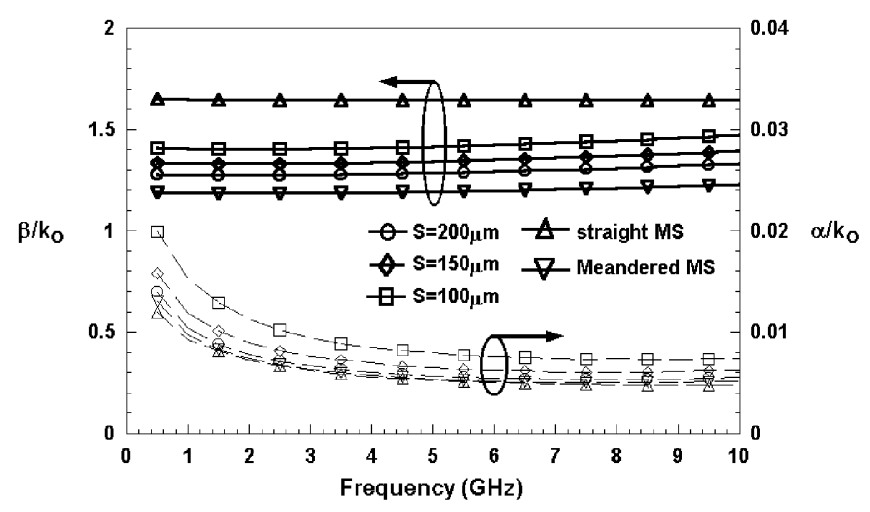

(a) The $\beta / k_{0}, \alpha / k_{0}$ vs. frequency

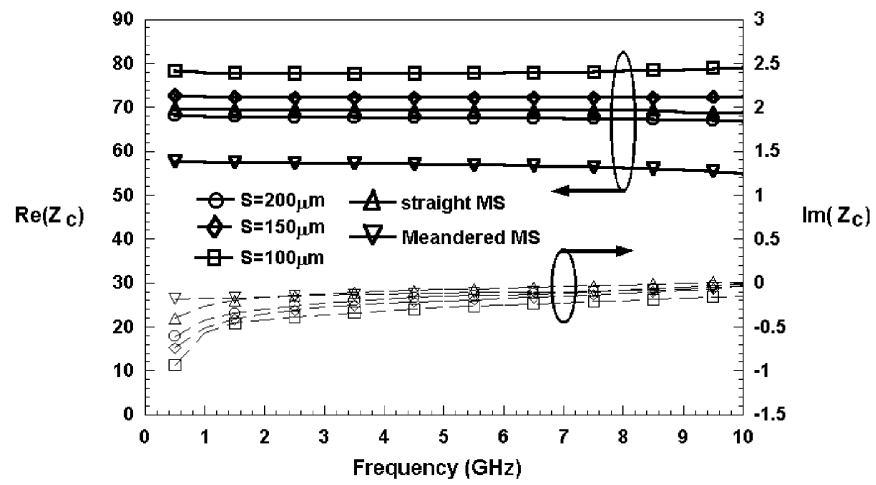

(b) The $\operatorname{Re}\left(Z_{c}\right), \operatorname{Im}\left(Z_{c}\right)$ vs, frequency

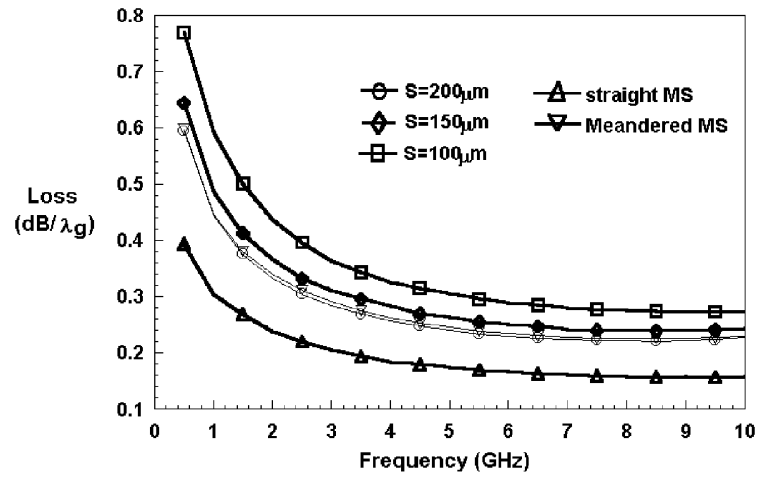

(c) The loss vs. frequency

Fig. 6. (a) Propagation characteristics $\left(\beta / k_{o}, \alpha / k_{o}\right)$, (b) complex characteristic impedance $\left[\operatorname{Re}\left(Z_{c}\right), \operatorname{Im}\left(Z_{c}\right)\right]$, and (c) loss per guided wavelength (decibels $\left./ \lambda_{g}\right)$ versus frequency of the 2-D CCS TL $(5 \times 4$ meander trace, the $W_{h}=300 \mu \mathrm{m}$ ) with various connecting arm widths $S$, and traditional MS and meandered MS with equal linewidths $W(=300 \mu \mathrm{m})$, total length $L(=9020 \mu \mathrm{m})$, and periodicity $P(=450 \mu \mathrm{m})$.

design of the CCS TL using $S$ as a control parameter for fine tuning the desired characteristic impedance to be used in Section IV for design of a rat-race hybrid.

The propagation characteristics, complex characteristic impedance, and the normalized attenuation in decibels per wavelength $\left(\mathrm{dB} / \lambda_{g}\right)$ versus frequency for the particular meandered CCS TL wandering in a $5 \times 4$ matrix with a ground opening of $300 \mu \mathrm{m} \times 300 \mu \mathrm{m}$ are shown in Fig. 6(a)-(c), respectively. For comparative purpose, the results of the straight MS and the meandered MS are also included in Fig. 6. The structural dimensions of all case studies investigated here are listed in the caption of Fig. 6.

Fig. 6(a) shows the normalized phase constant $\beta / k_{o}$ versus frequency, which are nearly flat between $0.5-10 \mathrm{GHz}$ for all case studies. The maximum variation of $\beta / k_{O}$ is smaller than 0.065 in Fig. 6(a) for the CCS TL with $S=100 \mu \mathrm{m}$. At $5.4 \mathrm{GHz}$, the corresponding values of $\beta / k_{o}$ are 1.64, 1.42, 1.35, 1.29, and 1.20 for a straight MS, meandered CCS TLs, and meandered MS, respectively. On the other hand, a decrease in the normalized attenuation constant $\alpha / k_{o}$ is noticeable between $0.5-3 \mathrm{GHz}$, and it flattens out from 3 to $10 \mathrm{GHz}$ for all case studies. The maximum value of the $\alpha / k_{o}$ is approximately 0.02 and 0.007 at 0.5 and $10 \mathrm{GHz}$, respectively for the CCS TL with $S=100 \mu \mathrm{m}$. The values of $\alpha / k_{o}$ are $0.008,0.006,0.006,0.005$, and 0.005 for meandered CCS TLs with $S=100,150$, and $200 \mu \mathrm{m}$, a meandered MS, and a straight MS at $5.4 \mathrm{GHz}$, respectively.

The left-hand-side axis of Fig. 6(b) plots the real part of $Z_{c}$ (characteristic impedance) versus frequency, and is also showing that all cases are relatively flat. Maximum variation of $\operatorname{Re}\left(Z_{c}\right)$ is only $3 \Omega$ for the meandered MS case investigated at extreme frequencies. The value of $\operatorname{Re}\left(Z_{c}\right)$ in descending order is approximately $78,72,70,68$, and $57 \Omega$ for the meandered CCS TL with $S=100$ and $150 \mu \mathrm{m}$, a straight MS, a meandered CCS TL with $S=200 \mu \mathrm{m}$, and a meandered MS, respectively, at $f_{o}=5.4 \mathrm{GHz}$. On the other hand, the imaginary part of $Z_{c}$ slowly increases and flattens out as operating frequency increases. The imaginary part of $Z_{c}$ is bounded in $(-0.95,0) \Omega$.

For purpose of clarity, we convert right-hand-side axis of Fig. 6(a) into loss in decibels per unit wavelength. The results are shown in Fig. 6(c). The maximum values of attenuation losses of the CCS TL with $S=100 \mu \mathrm{m}$ is 0.77 and $0.27 \mathrm{~dB} / \lambda_{g}$ at $f_{o}=0.5 \mathrm{GHz}$ and $10 \mathrm{GHz}$, respectively. Notice that the values of attenuation losses of CCS TLs with $S=150$ and $200 \mu \mathrm{m}$ are very close to those of the meandered MS.

The meandered CCS TL shows in all its aspects similar dispersion characteristics to those of a straight MS line. In practice, we treat the CCS TL like a quasi-TEM TL, although it is a 2-D guiding structure in nature.

\section{Miniaturized Rat-RACE Hybrid Using CCS TLs}

This section presents how CCS TLs are incorporated to designing a popular microwave rat-race hybrid that is often used in a power combiner/divider, balanced mixer/amplifier, etc. Following the design procedure of CCS TLs illustrated in Section III, one may choose the 70- $\Omega$ CCS TL by compromising the SWF, loss (decibels $/ \lambda_{g}$ ), current carrying capacity, and PCB design rules. The dashed line of Fig. 5(a) represents a 70- $\Omega$ characteristic impedance level, which intersects curves for a meandered MS, L7, L5, L4, L3, and L2. The design with larger dimensions in $S$ and $W$ will be less sensitive to process tolerance and capable of handling larger amount of currents. Therefore, L4 is a good choice. Fig. 5(b) and (c) further validates such a choice from the SWF and attenuation per wavelength points-ofview, both showing a good compromise among $Z_{c}, \mathrm{SWF}$, and losses. Taking the controlling parameters of L4, namely, $S=$ $150 \mu \mathrm{m}$ and $W_{h}=300 \mu \mathrm{m}$, we set $W=300 \mu \mathrm{m}$, as seen from Fig. 5(a). Next, we check such a set of selected parameters in Fig. 6(a)-(c) by varying the width of the connecting arm $S$ for the purpose of fine tuning. The results shown in Fig. 6 indicate that $Z_{c}$ can be $70 \Omega$ for $S$ between $150-200 \mu \mathrm{m}$. We select 


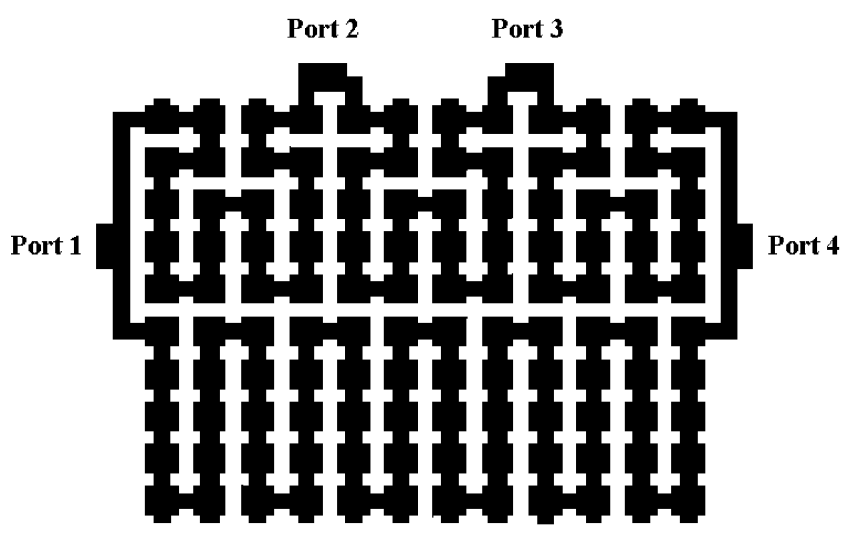

(a) The top pattern

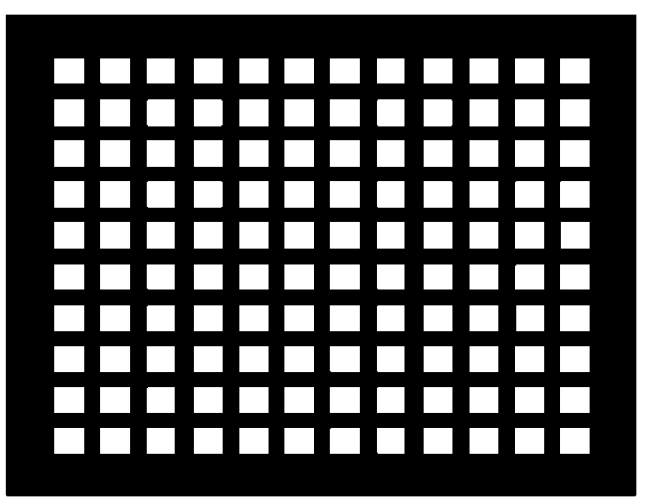

(b) The bottom pattern

Fig. 7. (a) Top pattern and (b) bottom pattern of the CCS rat-race hybrid coupler.

$S$ equal to $150 \mu \mathrm{m}$, contemplating that this is already a good choice for $Z c=72 \Omega$ under such a condition.

To this end, the design of the CCS TL for the rat-race hybrid is complete. What follows is an interesting part of the CCS TL circuit design. The entire rat-race coupler circuit is contained in a predetermined rectangular array of $10 \times 12$ cells without wasting any single grid. Notice that $\mathrm{T}$-junctions are required at the four ports. This can be designed by invoking field simulators for obtaining good electromagnetic models. Fig. 7(a) is the top layer metal of the CCS rat-race hybrid design, showing that three quarter-wavelength meandered CCS TLs are in series connections and tapped at ports 2 and 3 by T-junctions. Also shown in the lower half of Fig. 7(a) is the meandered CCS TL of three quarter-wavelengths in parallel with ports 1 and 4 . The bottom metal sheet of the CCS rat-race hybrid is shown in Fig. 7(b). The meshed ground plane is a 2-D periodic array with its etched portion complementing the top metallic layer structure.

The CCS rat-race hybrid is very small, with an area of only $5.85 \mathrm{~mm} \times 4.725 \mathrm{~mm}$, which is only $13 \%$ of that of the conventional MS ring-shape coupler. The available connectors, therefore, cannot be mounted directly on the rat-race hybrid. Strained by the available fixtures, we connect a semirigid coaxial cable to each port. The reference plane is then moved to the open end of the cable at each port by an open-short-load vector network analyzer (VNA) calibration procedure. Finally, ports 1-4 are soldered to the semirigid coaxial cables when needed for making good interfaces between coaxial connectors and the input/output ports of the CCS rat-race hybrid. The
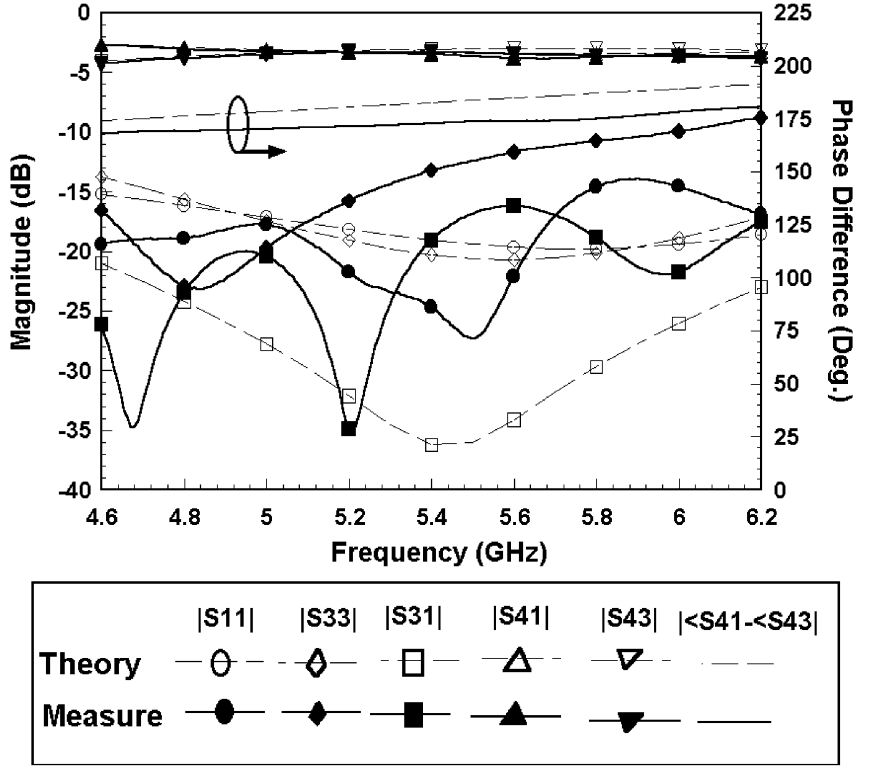

Fig. 8. Scattering parameters, return loss $\left|S_{11}\right|,\left|S_{33}\right|$, isolation $\left|S_{31}\right|$, coupling loss $\left|S_{41}\right|,\left|S_{43}\right|$, and phase difference $\left|\angle S_{41}-\angle S_{43}\right|$ versus frequency of the CCS rat-race hybrid prototype.

measured results are plotted in Fig. 8, superimposed by the theoretical plots between 4.6-6.2 GHz. The measured input reflection coefficients of ports 1 and 3 show better performance than the theoretical data and worse results at the high frequency side. The agreement, however, between theoretical results and measured data agree well and the measured response in its worst case is $-10 \mathrm{~dB}$ at $6.2 \mathrm{GHz}$, which is $0.8 \mathrm{GHz}$ from the design center frequency. The isolation between ports 1 and 3 is kept at least $+15 \mathrm{~dB}$ across the band. At $5.2 \mathrm{GHz}$, in particular, the measured isolation is over $35 \mathrm{~dB}$. The measured and simulated transmission characteristics $\left|S_{41}\right|$ and $\left|S_{43}\right|$ agree very well with each other, over the high-frequency band, deviating by approximately $0.3 \mathrm{~dB}$ for $\left|S_{41}\right|$ and $0.7 \mathrm{~dB}$ for $\left|S_{43}\right|$ from the theoretical values. The phase difference $\left|\angle S_{41}-\angle S_{43}\right|$ exhibits a $180^{\circ}$ phase reversal between ports 1 and 3 and the difference port (port 4 ), and the measured results are only $10^{\circ}$ lower than the theoretical results throughout the band.

\section{Distributed CMOS Oscillator USING CCS TLs}

Lumped $L C$ elements have been widely applied in monolithic RF CMOS and GaAs integrated circuits. The lumped elements operate in the frequency range in that the dimension of lumped elements is much smaller than the operational wavelength. This imposes a serious limit on using lumped elements for the state-of-the-art RFIC approaching millimeter waves and beyond. This section reports a basic research endeavour attempting to demonstrate that the distributed CCS TLs realized in CMOS technology can fully replace the lumped elements at no cost to chip area while achieving reasonable performance [29]. The target RF CMOS circuit is the 5.2-GHz differential cross-coupled oscillator whose $L C$ tank circuit will be substituted by the CCS TL. Referring to Fig. 9, transistors M1-M4 form two pairs of negative transconductance $(-g m)$ circuits in parallel. Between nodes $X$ and $Y$, the CCS TL resonates with the terminal parasitic capacitances associated with nodes $X$ 


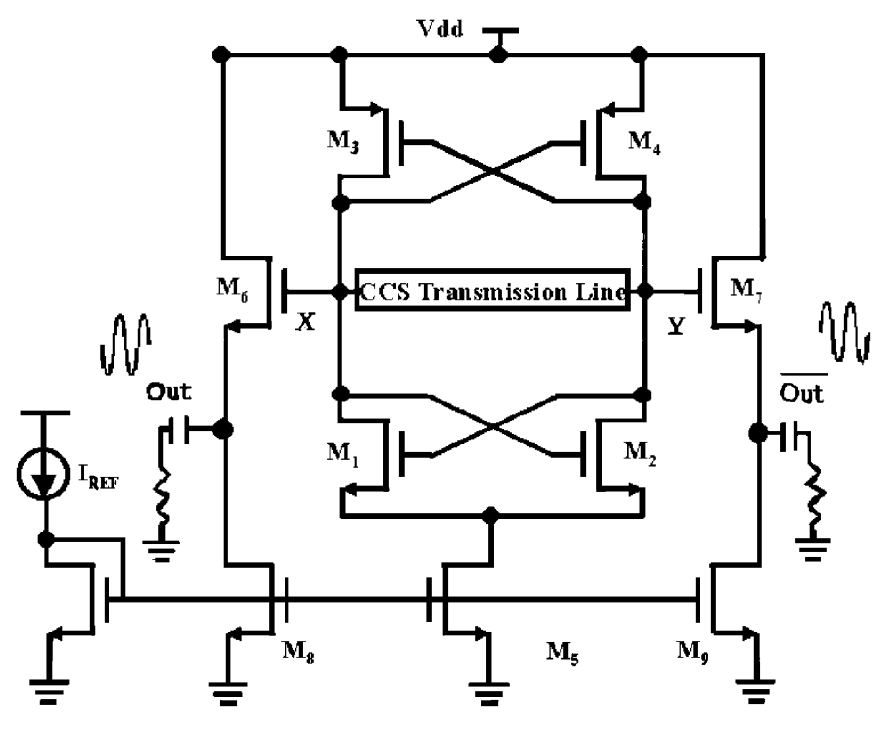

Fig. 9. CCS oscillator schematics.

and $Y$ at the desired frequency. When the negative $g m$ values can overcome the losses in the resonator, the circuit oscillates since $100 \%$ positive feedback signals are established through the cross-coupled wiring configuration. The parallel $L C$ circuit can be replaced by a half-wavelength TL if the parasitics at nodes $X$ and $Y$ are negligible. Consequently, when resonance occurs at the TL, nodes $X$ and $Y$ are out-of-phase, keeping the oscillator running differentially.

The first step for designing the CCS-based CMOS cross-coupled oscillator is the design of 50- $\Omega$ CCS TL. Circuit simulations show that CCS TL of different values of characteristic impedance can work well for the particular design. We choose a $50-\Omega$ line for convenience since many microwave circuits interface to external loads are referenced to $50 \Omega$. Fig. 10 shows the measured and simulated 50- $\Omega$ CCS TL based on the Taiwan Semiconductor Manufacturing Company (TSMC), Taiwan, R.O.C., 0.25- $\mu$ m 1P5M CMOS process technology, which has one poly resistor layer above the active devices and additional five metal layers stacked above the poly. Near the bottom metal layers, M1 and M2 are connected by vias to form thicker meshed ground plane and M4 and M5 (the top metal layer) are also connected by vias to form upper signal traces, thus forming the CCS guiding structure. These via connections reduce sheet resistance and improves the quality factor $(Q)$ of the CCS TL. The periodicity $(P)$ of the CCS TL is $15 \mu \mathrm{m}$. The upper surface has a rectangular patch of $W_{x}=10 \mu \mathrm{m}$ and $W_{y}=8 \mu \mathrm{m}$ and connecting arm of $S=3 \mu \mathrm{m}$. The lower complementary surface has a rectangular hole of $W_{h x}=14 \mu \mathrm{m}$ and $W_{h y}=10 \mu \mathrm{m}$. Fig. 10 compares the theoretical and measured propagation characteristics of the meandered CMOS CCS TL using the TSMC $0.25-\mu \mathrm{m}$ CMOS standard digital process. Excellent agreement of the measured and theoretical data in the real and imaginary parts of $Z_{c}$ (characteristic impedance) and losses in decibels $/ \lambda_{g}$ have been obtained. An on-wafer parasitic extraction procedure has been applied to alleviate the parasitic loading of probing pads, as well as the extra interconnects to the device-under-test. The results shown in Fig. 10 indicate that we have synthesized a $50-\Omega$ TL across

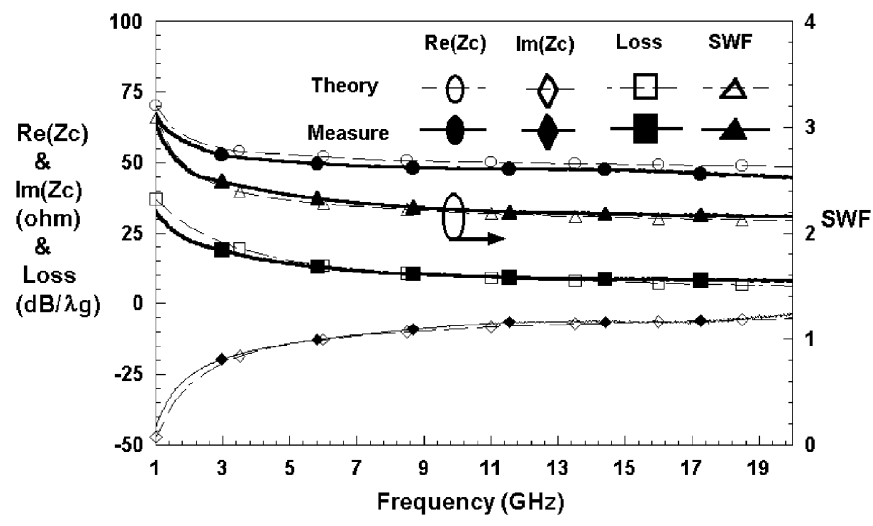

Fig. 10. Comparison of the theoretical and measured propagation characteristics $\operatorname{Re}(Z c), \operatorname{Im}(Z c)$, Loss (decibels $/ \lambda g$ ), and SWF of the meandered CMOS CCS TL obtained using a TSMC 0.25- $\mu \mathrm{m}$ 1P5M CMOS process with unit-cell structural parameters $P=15 \mu \mathrm{m}, S=3 \mu \mathrm{m}$, $W_{x}=10 \mu \mathrm{m}, W_{y}=8 \mu \mathrm{m}, W_{h x}=14 \mu \mathrm{m}$, and $W_{h y}=10 \mu \mathrm{m}$; 2-D CCS TL $(8 \times 3$ meander trace) and M5 and M4 are set into the top layer and M2 and M1 are set into the bottom layer. The theoretical data is simulated using IE3D.

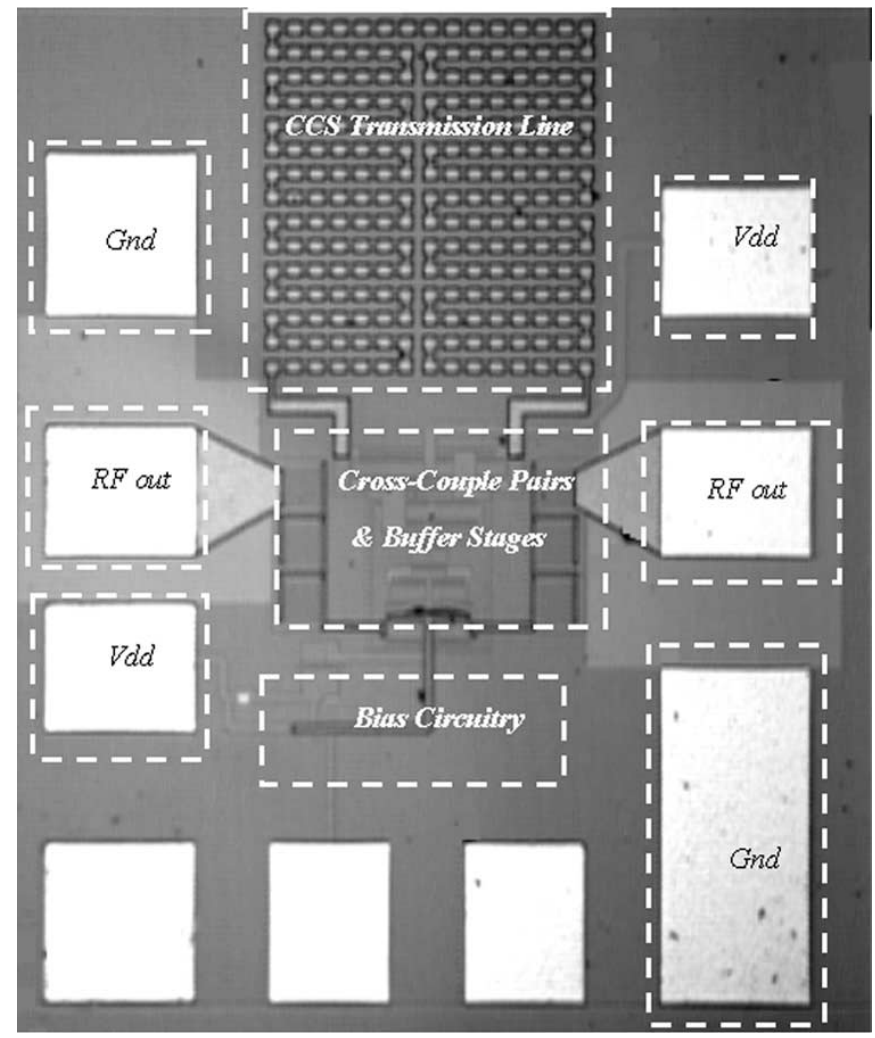

Fig. 11. 5.2-GHz CMOS cross-coupled oscillator based on a CCS TL resonator. Chip area: $0.5 \mathrm{~mm} \times 0.6 \mathrm{~mm}$ (including pads), $0.225 \mathrm{~mm} \times 0.21 \mathrm{~mm}$ (active area), and $0.225 \mathrm{~mm} \times 0.225 \mathrm{~mm}$ (CCS TL on top).

the $5-20-\mathrm{GHz}$ band. The SWF is approximately 2.2 across the band. The loss of $21.5 \mathrm{~dB} / \lambda_{g}$ is very high at $3 \mathrm{GHz}$. It declines to $15.3 \mathrm{~dB} / \lambda_{g}$ at $5 \mathrm{GHz}$ and reaching to $7.6 \mathrm{~dB} / \lambda_{g}$ at $15 \mathrm{GHz}$, reflecting the fact the CCS TL is a lossy TL at a low microwave frequency range. Thus, a one-half guided-wavelength $\lambda_{g}$ at $5.2 \mathrm{GHz}$ is $12.54 \mathrm{~mm}$. This length, at first sight, appears too long to be placed in a monolithic integrated circuit.

The CCS TL concept, however, can easily accommodate the half-wavelength TL resonator into a permissible area. Notice also that the corresponding $Q$ factor of the CCS TL reported 


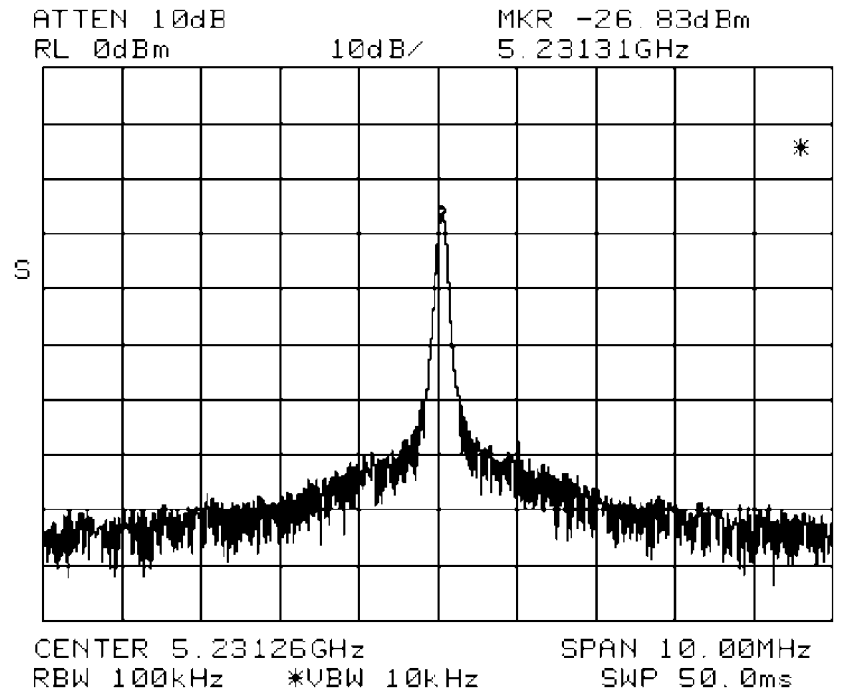

Fig. 12. Measured single-ended output power spectrum.

in Fig. 10 is approximately 1.84 at $5.2 \mathrm{GHz}$, which is not as impressive as a $Q$ factor. Nevertheless, if enough of the biased current source of M5 of Fig. 9 is applied, the resultant negative transconductance $(-g m)$ will be large enough to overcome the CCS TL losses, thus forming an oscillator that is free of $L C$ tank lumped elements. Fig. 11 shows a photograph of the cross-coupled CMOS oscillator that incorporates the CCS TL as the sole resonator for defining the oscillation frequency. When taking into account the parasitic capacitance at nodes $X$ and $Y$ of the oscillator shown in Fig. 9, the actual length of the meandered CCS TL is approximately $3.375 \mathrm{~mm}\left(\sim 0.135 \lambda_{g}\right.$ at $5.2 \mathrm{GHz}$ ), which still appears too big for monolithic integration. This is, however, not the case for the CCS TL integration. The CMOS chip photograph shows that the CCS TL resonator occupies only $225 \mu \mathrm{m} \times 225 \mu \mathrm{m}$ in chip area. The measured results at the attenuated output port of the oscillator are plotted in Fig. 12, which has a $0.208-\mathrm{pF}$ series capacitor connected to the buffered source follower of M6 or M7. The measurement shows that the free-running oscillation frequency is $5.23 \mathrm{GHz}$, which is only $40 \mathrm{MHz}$ deviated from the theoretical prediction based on the harmonic-balanced simulations. Such first-pass success has been witnessed for all eight samples evaluated. The operating current of this oscillator is $20 \mathrm{~mA}$ and the supply voltage is $3 \mathrm{~V}$.

\section{CONCLUSION}

This paper has proposed a new guiding structure of CCSs that provides a wider choice of characteristic impedance and flatter propagation characteristics than a meandered MS structure (see Fig. 5). It is especially appropriate for use in designing compact passive circuits using microwave TLs. The CCS TLs were used successfully in designing a $5.4-\mathrm{GHz}$ CCS rat-race hybrid prototype, the area required for which is only $13 \%$ of that of the conventional MS ring-shape coupler, using a PCB process. An $L C$-free fully integrated compact 5.2-GHz CMOS oscillator with an area totaling $500 \times 600 \mu \mathrm{m}^{2}$ including pads is designed. Its simulated and measured oscillation frequencies are within $1 \%$ of each other. More compact and further improved microwave integrated passive circuits, including CCS TLs, will be described in the near future.

\section{ACKNOWLEDGMENT}

The authors are grateful to H.-H. Wu, Industrial Technology Research Institute (ITRI), Hsinchu, Taiwan, R.O.C., for sharing his experience in IC fabrication.

\section{REFERENCES}

[1] C.-H. Lee, A. Sutono, S. Han, K. Lim, S. Pinel, E. M. Tentzeris, and J. Laskar, "A compact LTCC-based $K u$-band transmitter module," IEEE Trans. Adv. Packag., vol. 25, pp. 374-384, Aug. 2002.

[2] H.-S. Song and Y.-S. Lee, "A miniaturized $2.4 \mathrm{GHz}$ band multi-layer bandpass filter using capacitively loaded $\lambda / 4$ slow-wave resonator," in IEEE MTT-S Int. Microwave Symp. Dig., vol. 1, June 8-13, 2003, pp. $515-518$.

[3] S. Lee, J. Choi, G. S. May, and I. Yun, "Modeling and analysis of 3-D solenoid embedded inductors," IEEE Trans. Electron. Packag. Manufact., vol. 25, pp. 34-41, Jan. 2002.

[4] T. Nakatsuka, J. Itoh, S. Yamamoto, T. Yoshida, M. Nishitsuji, T. Uda, K. Nishii, and O. Ishikawa, "A highly miniaturized receiver front-end hybrid IC using on-chip high-dielectric constant capacitors for mobile communication equipment," in IEEE Microwave and Millimeter-Wave Monolithic Circuits Symp., 1995, pp. 85-88.

[5] H. Matsumoto, H. Ogura, and T. Nishikawa, "A miniaturized dielectric monoblock band-pass filter for $800 \mathrm{MHz}$ band cordless telephone system," in IEEE MTT-S Int. Microwave Symp. Dig., 1994, pp. 249-252.

[6] H. Matsumoto, T. Tsujiguchi, and T. Nishikawa, "A miniaturized dielectric monoblock duplexer matched by the buried impedance transforming circuit," in IEEE MTT-S Int. Microwave Symp. Dig., 1995, pp. $1539-1542$.

[7] R. K. Hoffmann, Handbook of Microwave Integrated Circuits. Norwood, MA: Artech House, 1987.

[8] Y. Ishikawa, T. Okada, S. Shinmura, F. Kanaya, K. Wakino, and T. Nishikawa, "A miniaturized low-spurious $1.9 \mathrm{GHz}$ MSW band-pass filter using YIG resonators with multi metal rings," in IEEE MTT-S Int. Microwave Symp. Dig., 1992, pp. 1403-1406.

[9] H. Ogawa and T. Itoh, "Slow-wave characteristics of ferromagnetic semiconductor microstrip line," IEEE Trans. Microwave Theory Tech., vol. MTT-34, pp. 1478-1482, Dec. 1986.

[10] F.-R. Yang, K.-P. Ma, Y. Qian, and T. Itoh, "A uniplanar compact photonic-bandgap (UC-PBG) structure and its applications for microwave circuits," IEEE Trans. Microwave Theory Tech., vol. 47, pp. 1509-1514, Aug. 1999.

[11] C.-K. Wu, H.-S. Wu, and C.-K. C. Tzuang, "Electric-magnetic-electric slow-wave microstrip line and bandpass filter of compressed size," IEEE Trans. Microwave Theory Tech., vol. 50, pp. 1996-2004, Aug. 2002.

[12] T. Tokumitsu, T. Hiraoka, H. Nakamoto, and T. Takenaka, "Multilayer MMIC using a $3 \mu \mathrm{m} \times 3$-layer dielectric film structure," in IEEE MTT-S Int. Microwave Symp. Dig., 1990, pp. 831-834.

[13] T. Hiraoka, T. Tokumitsu, and M. Aikawa, "Very small wide-band MMIC magic T's using microstrip lines on a thin dielectric film," IEEE Trans. Microwave Theory Tech., vol. 37, pp. 1569-1575, Oct. 1989.

[14] T. Tokumitsu, M. Hirano, K. Yamasaki, C. Yamaguchi, K. Nishikawa, and M. Aikawa, "Highly integrated three-dimensional MMIC technology applied to novel masterslice GaAs- and Si-MMIC's," IEEE J. Solid-State Circuits, vol. 32, pp. 1334-1341, Sept. 1997.

[15] K. Nishikawa, K. Kamogawa, K. Inoue, K. Onodera, T. Tokumitsu, M. Tanaka, I. Toyoda, and M. Hirano, "Miniaturized millimeter-wave masterslice 3-D MMIC amplifier and mixer," IEEE Trans. Microwave Theory Tech., vol. 47, pp. 1856-1862, Sept. 1999.

[16] C. Warns, W. Menzel, and H. Schumacher, "Transmission lines and passive elements for multilayer coplanar circuits on silicon," IEEE Trans. Microwave Theory Tech., vol. 46, pp. 616-622, May 1998.

[17] K. Wu, R. Vahldieck, J. L. Fikart, and H. Minkus, "The influence of finite conductor thickness and conductivity on fundamental and higher-order modes in miniature hybrid MIC's (MHMIC's) and MMIC's," IEEE Trans. Microwave Theory Tech., vol. 41, pp. 421-430, Mar. 1993.

[18] S. Banba and H. Ogawa, "Small-sized MMIC amplifiers using thin dielectric layers," IEEE Trans. Microwave Theory Tech., vol. 43, pp. 485-492, Mar. 1995. 
[19] T. Imaoka, S. Banba, A. Minakawa, and N. Imai, "Millimeter-wave wide-band amplifiers using multilayer MMIC technology," IEEE Trans. Microwave Theory Tech., vol. 45, pp. 95-101, Jan. 1997.

[20] W. P. Harokopus, Jr. and L. P. B. Katehi, "Electromagnetic coupling and radiation loss considerations in microstrip (M)MIC design," IEEE Trans. Microwave Theory Tech., vol. 39, pp. 413-421, Mar. 1991

[21] N. Jain and P. Onno, "Methods of using commercial electromagnetic simulators for microwave and millimeter-wave circuit design and optimization," IEEE Trans. Microwave Theory Tech., vol. 45, pp. 724-746, May 1997.

[22] A. K. Agrawal and G. F. Mikucki, "A printed circuit hybrid-ring directional coupler for arbitrary power divisions," in IEEE MTT-S Int. Microwave Symp. Dig., 1986, pp. 139-142.

[23] R. Levy and L. F. Lind, "Synthesis of symmetrical branch-guide directional couplers," IEEE Trans. Microwave Theory Tech., vol. MTT-16, pp. 80-89, Feb. 1968

[24] C. Quendo, E. Rius, C. Person, and M. Ney, "Integration of optimized low-pass filters in a bandpass filter for out-of-band improvement," IEEE Trans. Microwave Theory Tech., vol. 49, pp. 2376-2383, Dec. 2001.

[25] T. Itoh, "Generalized spectral domain method for multiconductor printed lines and its applications to tunable suspended microstrips," IEEE Trans. Microwave Theory Tech., vol. MTT-26, pp. 983-987, Dec. 1978.

[26] H. Kamitsuna, "A very small, low-loss MMIC rat-race hybrid using elevated coplanar waveguides," IEEE Microwave Guided Wave Lett., vol. 2, pp. 337-339, Aug. 1992.

[27] W. R. Eisenstadt and Y. Eo, " $S$-parameter-based IC interconnect transmission line characterization," IEEE Trans. Comp., Hybrids, Manufact. Technol., vol. 15, pp. 483-490, Aug. 1992.

[28] K. C. Gupta, R. Garg, I. Bahl, and P. Bhartia, Microstrip Lines and Slotlines. Norwood, MA: Artech House, 1996, pp. 189-194.

[29] C.-K. C. Tzuang, C.-C. Chen, and W.-Y. Chien, " $L C$-free CMOS oscillator employing two-dimensional transmission line," in Proc. IEEE Int. Frequency Control Symp. and PDA Exhibition/17th Euro. Frequency and Time Forum, 2003, pp. 487-489.

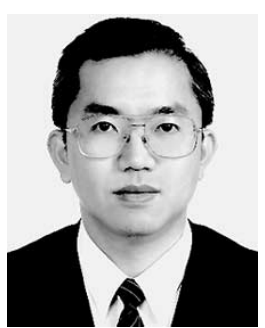

Chih-Chiang Chen (S'97) was born in Taipei, Taiwan, R.O.C., on June 17, 1963. He received the B.S.E.E. and M.S. degrees in electronic engineering from the Chung Cheng Institute of Technology, Tai-Hsi, Taiwan, R.O.C., in 1988 and 1992, respectively, and is currently working toward the Ph.D. degree at the National Chiao Tung University, Hsinchu, Taiwan, R.O.C

From 1988 to 1989, he was a Teaching Assistant with the Chung Cheng Institute of Technology, where he was involved in the design of electronic circuits. From 1993 to 1996, he was with the Chung Shang Institute of Science and Technology, San-Hsia, Taiwan, R.O.C., where he was involved in the engineering development and automatic testing system (ATS). His current research interests include microwave, millimeter-wave integrated-circuit, and planar antenna design.

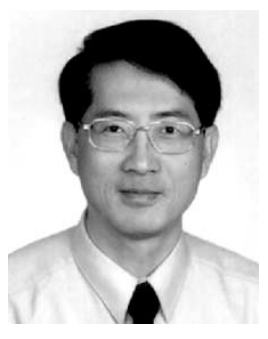

Ching-Kuang C. Tzuang (S'80-M'80-SM'92F'99) received the B.S. degree in electronic engineering from the National Chiao Tung University, Hsinchu, Taiwan, R.O.C., in 1977, the M.S. degree from the University of California at Los Angeles, in 1980, and the Ph.D. degree in electrical engineering from the University of Texas at Austin, in 1986.

From 1981 to 1984 , he was with TRW, Redondo Beach, CA, where he was involved with analog and digital MMICs. Since 1986, he has been with the Institute of Communication Engineering, National Chiao Tung University. In February 2004, he joined the Graduate Institute of Communication Engineering, Department of Electrical Engineering, National Taiwan University, Taipei, Taiwan, R.O.C., where he continues his research on advanced guiding structures for research and development of RF system-on-a-chip, integrating active and passive microwave/millimeter-wave $\mathrm{RF}$ signal-processing components into a single chip. His research activities also involve the design and development of millimeter-wave and microwave active and passive circuits and the field theory analysis and design of various complex-waves guiding structures and large-array antennas. He has supervised 61 M.S. students and $19 \mathrm{Ph} . \mathrm{D}$. students.

Dr. Tzuang helped in the formation of the IEEE Microwave Theory and Techniques Society (IEEE MTT-S) Taipei Chapter, and served as secretary, vice chairman, and chairman in 1988, 1989, and 1990, respectively. He has been on the Asia-Pacific Microwave Conference International Steering Committee, where, since 1994, he has represented the Taipei Chapter as the international liaison officer. 